\title{
Special issue on information systems quality management in practice
}

\author{
Martin Shepperd ${ }^{1} \cdot$ Fernando Brito e $\mathrm{Abreu}^{2} \cdot$ Ricardo Perez-Castillo $^{3}$
}

Published online: 15 February 2022

๑ The Author(s), under exclusive licence to Springer Science+Business Media, LLC, part of Springer Nature 2022

\section{Introduction to the special issue}

The business core of today's companies is increasingly based on information technology. Indeed, through digital transformation, companies are offering new and disruptive products and services based on new ways of managing information alongside enterprise information systems (EIS). In this context, quality deserves a special mention, since quality of data and software, among other EIS artefacts, is key. It helps ensure that poor quality does not prevent achieving the intended business goals of the EIS and, thereby, hindering competitive advantage in a changing world. Although there are some advances to managing quality in EIS, it is usually perceived that there is a gap between the methods and techniques proposed by academia and what is used by practitioners.

This special issue comprises 10 papers that present innovative and significant findings and experiences in the field of quality management practices; methods and techniques in use by academia and industry that can serve to reduce the gap between companies' needs and the status quo, and finally, how researchers and practitioners employ such methods and techniques in practice.

Digkas et al. analyse the temporality of code technical debt introduction in new code, i.e. whether the introduction of technical debt is stable across the lifespan of the project, or if its evolution presents spikes. Through the analysis of several open-source projects, this study investigates the relation of technical debt introduction to the development team's workload in a given period, as well as to the experience of the development team.

Siebert et al. provide a quality model for machine learning systems that allow practitioners to specify and assess qualities for these systems objectively. This quality model defines measurable quality properties that can be evaluated in practice.

Ricardo Perez-Castillo

ricardo.pdelcastillo@uclm.es

Martin Shepperd

martin.shepperd@brunel.ac.uk

Fernando Brito e Abreu

fba@iscte-iul.pt

1 Brunel University London, Uxbridge, UK

2 ISCTE-IUL, Lisbon, Portugal

3 University of Castilla-La Mancha, Talavera de la Reina, Spain 
There are three articles about quality management in practice for IoT environments. First, Niedermaier et al. present an approach to define and evaluate the dependability of complex IoT systems through a model based on ISO/IEC 25040. Second, Chehida et al. provide an approach for a formal specification and analysis of sensor behaviour starting from existing sensor traces. Third, Olianas et al. present MATTER, a tool for generating end-to-end IoT test scripts.

Regarding software verification and validation, the special issue includes two articles. Soto Sánchez et al. provide a dataset for end-to-end tests in web applications. This dataset attempts to address some problems found in this kind of test, such as the infrastructure required for running them, the complexity of the setup and the lack of proper characterization of the faults and their fixes. This is complemented by Stray et al. who present a study that analyses some human factors of the agile software tester.

The special issue also includes articles that address quality topics in a broader range of applications. For example, Malgaonkar et al. develop and evaluate a novel approach based on natural language processing, feature engineering and word sense disambiguation to generate a dynamic taxonomy that groups related app reviews. Varshney and Ujjwal propose a novel model named Secure Virtual Infrastructure Model (SViM) to enhance the service quality, accessibility and usability of the IaaS cloud. Finally, looking to the future, PérezCastillo et al. propose QRev, a technique for migrating Q\# code toward hybrid (classicquantum) information systems based on the Knowledge Discovery Metamodel (ISO/IEC 19506).

As guest editors, we thank all the authors for their valuable contributions, the referees who reviewed the papers in this issue and to Rachel Harrison (Software Quality Journal Editor-in-Chief) for her continuous support.

Publisher's Note Springer Nature remains neutral with regard to jurisdictional claims in published maps and institutional affiliations. 